\title{
Model for growth and morphology of fungal mycelium
}

\author{
Bhagyashri Shinde, Shagufta Khan, and Sudipto Muhuri $\odot^{*}$ \\ Department of Physics, Savitribai Phule Pune University, Pune, India
}

(Received 3 November 2019; accepted 23 March 2020; published 30 April 2020)

\begin{abstract}
We present a minimal driven lattice gas model which generates the morphological characteristics associated with single-colony mycelium arising from the growth and branching process of fungal hyphae, which is fed by a single source of nutrients. We first analyze the growth and transport process in the primary hypha modeled as a growing one-dimensional (1D) lattice, which is subject to particle (vesicle) loss due to the presence of dynamically created branching sites. We show that the spatial profile of vesicles along the growing lattice is an exponential distribution, while the length grows logarithmically with time. We also find that the probability distribution of length of the hypha tends to a Gaussian distribution function at late times. In contrast, the probability distribution function of the time required for growth to a specific length tends to a broad log-normal distribution. We simulate the resultant 2D morphology generated by the growing primary hypha, quantifying the motility behavior and morphological characteristics of the colony. Analysis of the temporal behavior and morphological characteristics of the resultant 2D morphology reveals a wide variability of these characteristics, which depend on the input parameters which characterize the branching and elongation dynamics of the hyphae. By calibrating the input parameters for our model, we make some quantitative comparisons of the predictions of our model with the observed experimental growth characteristics of fungal hyphae and the morphological characteristics of single-colony fungal mycelium.
\end{abstract}

DOI: 10.1103/PhysRevResearch.2.023111

\section{INTRODUCTION}

Fungi are an integral part of the nutrient cycle in ecosystems and they serve as important model systems for genetic research due to their relative simplicity as an eukaryotic cellular system $[1,2]$.

Fungi exhibit a filamentous growth process. The basic filament structure of fungi-the hyphae-grows by means of extension and branching at the tip, giving rise to a multicellular complex network, i.e., the mycelium [1,3]. In order to develop a comprehensive understanding of the morphology of the entire mycelium, it is pertinent to develop an understanding of the transport and branching process of the individual fungal hypha and the morphological features of the colony formed as a result of such a growth process. Depending on the scale of description of the growth phenomenon, theoretical models for growth and transport in individual hypha and fungal colonies belong to the categories of tip-scale models [4,5], intermediate-scale models [6-9], and macroscale models $[2,10]$. The tip-scale models such as the vesicle supply center (VSC) model focus on aspects of the extension and shape of the hyphal tip [4,5], and the typical length scales of the description are $\sim 100 \mu \mathrm{m}$. For this model, the connection of the growth or branching process with the process of vesicle

\footnotetext{
*sudipto@physics.unipune.ac.in

Published by the American Physical Society under the terms of the Creative Commons Attribution 4.0 International license. Further distribution of this work must maintain attribution to the author(s) and the published article's title, journal citation, and DOI.
}

supply from the subapical region of the fungi is not explicitly taken into account $[8,9]$. On the other hand, the macroscale models focus on a description in terms of effective interaction of the fungal colony as a whole with the environment, without explicitly taking into account the metabolic processes at play for the individual hypha that constitute the colony $[10,11]$. The model that we study belongs to the category of intermediate-scale description, wherein we focus on a motordriven process of growth and branching in individual hyphae which is fed by a single source of nutrients and generates a single-colony mycelium. Much of the previous work in the domain of such intermediate-scale description of fungal growth has taken recourse to continuum models and focused on the details of biomechanics, without explicitly considering the role of molecular motors $[9,10]$. In contrast, we investigate the role of molecular motors using a model of driven lattice gas.

Driven lattice gas models have provided a useful description for the plethora of biological processes encompassing transport across biomembranes [12], dynamics of ribosomes in m-RNA [13], motor-driven intracellular transport [14-18], transport in fungal hyphae $[8,19,20]$, and other driven phenomenon [20-29]. In particular, a driven lattice gas model, i.e., the dynamically extending exclusion process (DEEP), has been adopted for modeling the extension of fungal hyphae $[8,19]$. For DEEP, particles hop unidirectionally on the lattice, interacting with other particles via hard-core repulsion, and the particles at the growing end of the lattice dynamically create a new lattice site, leading to the overall extension of the lattice $[8,19]$. While this model has been able to relate the growth of the fungal hyphae with the supply of nutrients, one inadequacy has been that the process of branching and 
related loss of the transported particles has not been taken into account. For instance, for aerial hyphae of sporangiophore, branching is indeed observed. Further, the elongation process of the individual hypha is such that there is a slow down of their growth rates as their length increases [9,30]. This is in contrast to the results obtained for DEEP, which predicts a growth rate of the hypha that is uniform, without any slowdown $[8,19]$.

Our minimal model is a generalization of the dynamically extending exclusion process (DEEP), encompassing the process of both linear growth and branching. Also, in contrast to continuum biomechanical models [9], which involve many parameters, our coarse-grained description involves very few input parameters, e.g., nutrient supply rate, growth rate at the tip, branching rate, and flow rate of nutrients to the different branches of the growing lattice. One experimental system that serves as a paradigmatic example is the fungus Neurospora crassa [31,32]. For this system, the material necessary for growth of the hyphae is packaged as vesicles and supplied by a single source located in the subapical region of the fungi $[31,32]$. The transport of these vesicles is done by molecular motors, which walk along the parallel array of microtubule filaments, carrying these vesicles to the tip of the hypha $[33,34]$. In the apical region of the tip, the intracellular organelle Spitzenkorper is involved in the synthesis of new cell wall from the vesicles, leading to growth [31]. Apart from the linear elongation process of the hyphae, the lateral branching process of the fungal hyphae is also observed $[9,30,32]$. The process of branching and elongation of a single primary hypha, fed by a single source of nutrient, generates a singlecolony mycelium. We study the morphological characteristics and growth pattern of such colonies on a surface using a twodimensional (2D) minimal model. Two-dimensional growth processes have been studied for randomly branched polymers [35-37] and growth of cell colonies [38]. In this context, variants of lattice animal models such as the Eden Aggregation process have been used to characterize the growth processes on discrete lattices [38-44], while continuum models such as Flory Theory for branched polymers have been developed to study the polymerization process under different conditions [35-37]. The connections between these discrete lattice animal models with the polymerization models have also been studied [35-37,45,46]. There is, however, one crucial distinction of these models with the model that we study in this article. In particular, for our model, the elongation and branching process at individual sites of the colony is coupled to the supply of nutrients at those sites, and is in contrast to the growth process of branched polymers and lattice animals for which the growth process at the sites is not subject to such a constraint.

In Sec. II, we first describe the 1D minimal model for the growth and transport in the primary hypha, specifying the dynamical rules for growth, and set up the corresponding equations of motion for the system. Further, we also specify the dynamical rules governing the development of the 2D mycelium arising from the branching process of the hyphae.

In Sec. III, we obtain the mean-field (MF) analytical solutions of the length of the individual primary hypha as a function of time as well as the spatial profile of the transported cargo along the hypha. We then compare these results with the Monte Carlo (MC) simulation results. By performing MC simulations, we also obtain the probability distribution of lengths of the primary hypha, and the probability distribution of the time required for growth up to a specified length of the hypha.

In Sec. IV, we discuss the results for the spatial and temporal features associated with single-colony mycelium that is generated from the elongation and branching process of the primary hyphae. In Sec. V, we calibrate the input parameters of the model to reproduce the quantitative measures associated with the growth characteristics of the individual fungal hypha and single-colony mycelium, which are observed in experiments. In Sec. VI, we summarize our results and discuss the insights gained from the findings of this model in understanding the growth and morphological characteristics of single-colony mycelium.

\section{MODEL}

In general, the process of linear growth and lateral branching (in the same plane) of the primary fungal hypha would generate a 2D morphology. We first describe the 1D model for the growth and transport behavior of the primary hypha. Subsequently, we would discuss the generalized model for describing the 2D morphology of the hyphal colony emerging due to the lateral branching process and linear extension of the fungal hyphae.

\section{A. Effective 1D model for growth for primary hypha}

We consider the initial state of the fungal hypha to be an object of 1D spatial extension with an initial specified length $\epsilon$ and which we refer to as the primary hypha. From the perspective of the linear extension of this primary hypha, the effect of branching manifests as a process of material loss along the hyphae during the process of its growth. We represent this growing primary fungal hypha as a 1D discrete lattice. At any instant of time $t$, the total number of lattice sites is $N$, corresponding to a length $L=\epsilon N$ (Fig. 1). Vesicles required for growth are represented by particles, which are transported by molecular motors along the lattice from left to right. First, we describe the dynamics of the particles at the lattice boundaries. At the left end of the lattice, at site $i=1$, particles enter the lattice with a rate $\alpha$ provided the boundary site is vacant. At the boundary site on the right, at site $i=N$, which corresponds to the tip of the hypha, two processes can occur as follows: (a) If the tip site at $i=N$ is occupied by a particle, then with a rate $\gamma$ an additional empty lattice site to the right is created, and the site $i=N$ also become empty. This process leads to overall increment of the length of the lattice from $L$ to $L+\epsilon$, while the total number of lattice sites is incremented from $N$ to $N+1$. This dynamics of lattice growth is exactly the same as that of DEEP [8,19]. (b) With a rate $1-\gamma$, an occupied tip site $i=N$ not only creates a new empty lattice site to the right, but the lattice at $i=N$ becomes an empty defect site. The overall extension rate of the lattice is 1 . These defect sites correspond to the branching sites that would get created dynamically. 


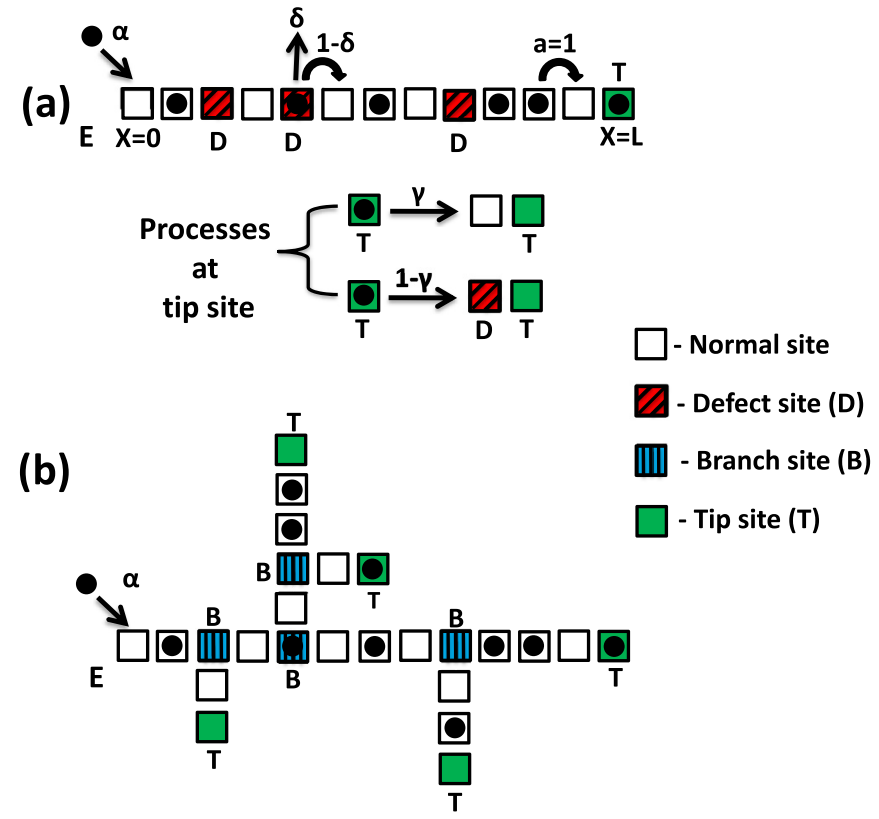

FIG. 1. (a) Schematic of the dynamical processes for growing primary hypha represented as a 1D growing lattice. With a rate $\gamma$, an occupied tip site (T) becomes two normal empty sites, while with rate $1-\gamma$ two empty lattice sites are created, with the preceding site transforming into a defect site (D). The particle entry rate at the first site $(\mathrm{E})$ is $\alpha$, the particle hopping rate is 1 for a normal (nondefect) site, and the loss rate of particles at the defect site is $\delta$. (b) Single-colony mycelium represented as a 2D lattice with entry rate $\alpha$, branch site creation rate $1-\gamma$, hopping rate of 1 for a normal (nonbranching) site, and particle flow rate to secondary branch $\delta$. For the branching sites, the hopping rate on the same branch is $1-\delta$ and the overall growth rate at the tip is 1 . Here, E denotes the site of entry, $\mathrm{T}$ denotes the tip site, and $\mathrm{B}$ denotes a branching site.

If one considers the full 2D morphology resulting from this growth process, then the defect sites would correspond to the branching sites for which the vesicle supply from the static end of the hyphae would be split between the two branches, leading to eventual growth of these two separate branches. However, from the perspective of the primary hypha, the effect of branching would manifest as a loss of vesicle at the defect sites. Thus, effectively, at any instant of the time, the 1D lattice, representing the primary hypha, would be comprised of bulk normal bulk sites $(\mathrm{N})$, defect sites (D), apart from the boundary sites at the growing tip (T), and boundary site at the nongrowing end (E), which corresponds to the site where particles enter the lattice. Particles in the bulk of the lattice interact with each other via excluded volume effect, thus restricting the maximum occupancy per lattice site to 1 . For any normal bulk site $i$, the particles hop unidirectionally to the adjacent site to the right, with rate 1 provided the site at $i+1$ is vacant. At the defect site, with a rate $\delta$, there is a loss of particle from the site, while with a rate $1-\delta$, the particles hop to the site $i+1$, provided it is empty. This completes the specification of the dynamics of the 1D model. All the dynamic processes for this dynamic lattice model are schematically depicted in Fig. 1(a).

\section{B. 2D model for single-colony mycelium}

We consider the growth process of a single colony on a square lattice, arising due to the branching and elongation process of the fungal hyphae constituting the colony. We consider an initial starting configuration of the colony which is a fungal hypha and is represented by a 1D lattice of length $\epsilon$ placed along the $x$ axis at the origin. The processes of particle entry at the left boundary are the same as that of the 1D model, with the particles entering at the left end of the lattice located at $(0,0)$, with a rate $\alpha$ provided the site is vacant. If there is particle at the tip site, the following two processes occur: (i) Pure linear extension. With a rate $\gamma$, a new lattice site is created in the original direction of movement of the particle, provided that the site is not occupied by the colony itself. The underlying assumption made here is that the presence of the colony obstructs and arrests the linear growth process of a hyphal tip. (ii) Branching and extension. With a rate $1-\gamma$, two new empty lattice sites are created - an empty lattice in the original direction of movement of the particle and an empty site perpendicular to it. One of two perpendicular directions is chosen randomly with equal probability. Again this process is subjected to the constraint that it occurs only if the adjacent site in the direction of growth and the adjacent sites in the perpendicular direction are not occupied by the colony itself. Such branching process would transform the tip site (T) at time $t$ into a branching site (B) at time $t+1$. It would also create two new tip sites in the direction of original movement of the particle and in a direction perpendicular to it. Both of these processes would lead to growth of the single colony comprised of these hyphae. Thus, at any instance of time, any site in the bulk of the colony would either be a branching site (B) or a normal site N (a site without any branching). At any instance, a particle on a normal site will hop unidirectionally with rate 1 to the adjacent site in the original direction of motion of the particle provided it is empty. For a particle on a branching site, with a rate $\delta$, the particle would hop onto the other branch site provided the adjacent site on the branch is empty, while with rate $1-\delta$, it would continue to hop to the adjacent site in the original direction of movement provided that site is empty. A typical configuration is schematically depicted in Fig. 1(b).

\section{MC simulation of the process}

To determine the density and current profiles on hypha, Monte Carlo (MC) simulations have been performed to simulate the various processes for the 1D model described in Sec. II A using the procedure of random-sequential update of the sites $[47,48]$. In this procedure, at any given time step, a site is chosen at random with equal probability and the lattice site is updated according to the rules of dynamics specified in Sec. II A. Each Monte Carlo unit of time, $\Delta t_{m c}$, corresponds to an interval of real time $\Delta t$, such that $\Delta t=\Delta t_{m c} / N(t)$. We note that since the lattice length is growing, therefore $N$ changes with time. For simulating the configurations for the 1D model, the initial starting configuration is a 1D lattice with number of sites $(N=1)$, which is occupied by a particle at $x=0$. For the $2 \mathrm{D}$ model, the initial starting configuration is also a 1D lattice with a particle occupying the site specified by coordinates $x=0, y=0$, and the initial growth direction 
being along the $+x$ direction. We adopt the synchronous update procedure (also referred to as the fully parallel update procedure) for updating the lattice sites for the 2D model [47-49]. For this update procedure, at any instance of time $t$, for a given configuration, all the lattice sites are updated simultaneously according to the rules of dynamics specified in Sec. II B [47]. One distinct advantage of performing MC simulations using the synchronous update procedure is that it is relatively faster than the random-sequential update procedure [47] and has been used in the context of modeling traffic flow [48]. Ensemble averaging is done typically for 10000 samples for the 1D model and for 100 samples for the 2D model, starting with the same initial configuration. For the 1D model, we have additionally also simulated the growing lattice using a fully parallel update procedure to characterize the temporal behavior of the growing lattice and compared the simulation results with the one obtained using a randomsequential update procedure.

\section{CHARACTERISTICS OF SINGLE HYPHA}

Having described the 1D model for growth of the primary hypha in Sec. II A, we now discuss the growth characteristics that we obtain for this minimal model.

\section{A. Survival probability and density profile}

For the 1D model for hyphal growth, we consider an ensemble of a similarly prepared system. We define survival probability $P(i \mid j)$ (with $i>j$ ) as the probability of a particle in site $j$ to reach site $i$ without leaving the 1D lattice,

$$
P(i \mid j)=\prod_{s=j}^{i-1} P(s+1 \mid s) P(j),
$$

where $P(s+1 \mid s)$ is the conditional probability for a particle at site $s$ to reach site $s+1$, and $P(j)$ is the probability of occupancy of site $j$.

On averaging over the ensemble, the average survival probability for a particle at site $s$ to reach a site $s+1,\langle P(s+1 \mid s)\rangle$ can be expressed in terms of the probability of occurrence of defect/normal sites and their respective survival probabilities as follows:

$$
\langle P(s+1 \mid s)\rangle=P_{N}(s+1 \mid s) P(N)+P_{D}(s+1 \mid s) P(D),
$$

where $P(D)$ is the probability that the site $s$ is a defect site, $P(N)$ is the probability that it is a normal site, $P_{D}(s+1 \mid s)$ is the survival probability of the particle at site $s$ to reach site $s+1$ if the site $s$ is a defect site, and $P_{N}(s+1 \mid s)$ is the survival probability of the particle at site $s$ to reach site $s+1$ if the site $s$ is a normal site. It therefore follows that $\langle P(s+1 \mid s)\rangle=$ $\gamma+(1-\delta)(1-\gamma)$, and the expression for average survival probability $\langle P(i \mid j)\rangle$ assumes the form

$$
\langle P(i \mid j)\rangle=P(j)[1-\delta+\delta \gamma]^{(i-j)} .
$$

The distance of separation between site $i$ and site $j$ in terms of lattice spacing $\epsilon$ can be expressed as $x=(i-j) \epsilon$. Then the expression for the average probability of a particle surviving a distance $x$ is

$$
P(x)=\exp \left(\frac{K x}{\epsilon}\right)
$$

where $K=\ln [1-\delta+\delta \gamma]$. The steady-state average occupancy $\rho(0)$ at the left end of the lattice at $x=0$ is $\alpha$.

When the excluded volume effect is ignored for the particle hopping process in the growing lattice, the steady-state average occupancy $\rho(x)$ may be expressed as $\rho(x)=\rho(0) P(x)$, and it follows from Eq. (4) that the approximate expression for the average occupancy $\rho(x)$ is

$$
\rho(x)=\alpha \exp \left(\frac{K x}{\epsilon}\right) .
$$

Alternatively, the approximate steady-state expression of $\rho(x)$ in Eq. (5) can also be obtained by writing the evolution equation for mean occupancy $[15,16]$, when the excluded volume effects are ignored. Denoting $\left\langle n_{i}\right\rangle=\rho_{i}$ as the mean occupancy at the $i$ th site, the evolution equation then reads

$$
\begin{aligned}
\partial_{t} \rho_{i}= & \gamma \rho_{i-1}+(1-\gamma)(1-\delta) \rho_{i-1} \\
& -\gamma \rho_{i}-(1-\gamma)(1-\delta) \rho_{i}-\delta(1-\gamma) \rho_{i} .
\end{aligned}
$$

The corresponding steady-state condition $\partial_{t} \rho_{i}=0$ leads to the relation $\rho_{i}=(1-\delta+\delta \gamma) \rho_{i-1}$. Along with the boundary condition $\rho_{1}=\alpha$, this leads to the expression of $\rho(x)$ which is identical to Eq. (5).

Including the excluded volume effect within a MF approximation $[15,16]$, where we factorize the two-point correlators arising out of the product of occupation numbers of neighboring sites, would lead to an evolution equation of the form

$$
\begin{aligned}
\partial_{t} \rho_{i}= & \gamma \rho_{i-1}\left(1-\rho_{i}\right)+(1-\gamma)(1-\delta) \rho_{i-1}\left(1-\rho_{i}\right) \\
& -\gamma \rho_{i}\left(1-\rho_{i+1}\right)-(1-\gamma)(1-\delta) \rho_{i}\left(1-\rho_{i+1}\right) \\
& -\delta(1-\gamma) \rho_{i} .
\end{aligned}
$$

The interpretation of the terms on the right are as follows: The first term corresponds to a gain term due to a particle from a normal site at position $i-1$ hopping to site $i$, the second term is due to a particle at defect site at $i-1$ hopping to site $i$, the third and fourth terms are the terms associated with particle hopping out of site $i$ to site $i+1$ from a normal and defect site, respectively, while the last term is associated with a particle leaving the lattice from a defect site. The corresponding steady-state solution in the continuum limit (with $x=i \epsilon$ ) may be obtained [14,16]. Ignoring terms of the order of $\epsilon^{2}$ leads to an implicit solution of $\rho(x)$,

$$
2(\rho-\alpha)-\ln \left(\frac{\rho}{\alpha}\right)=\left(\frac{x}{\epsilon}\right)\left[\frac{\delta(1-\gamma)}{1-\delta+\delta \gamma}\right] .
$$

In Fig. 2(a), we show a comparison of the spatial profile of the average occupancy along the growing 1D lattice obtained by approximate analytical means, e.g., Eq. (5) and Eq. (8) with the profile obtained by MC simulations.

\section{B. Temporal behavior of hyphal length}

For our model, we have set the rate of the overall growth rate of the lattice to 1 unit per unit time. This corresponds to a growth rate of $\epsilon$ per unit time, when the tip site is occupied by a particle. Thus the mean linear growth rate of the length of 

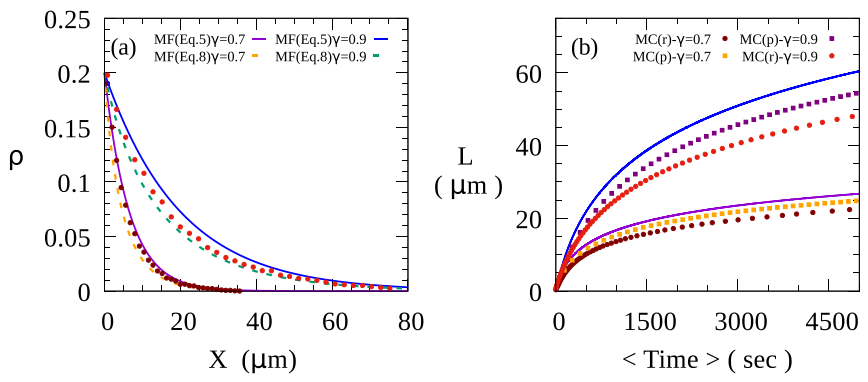

FIG. 2. (a) Average occupancy of particles $(\rho)$ as a function of distance from the site of particle entry into the lattice $(x)$ for the 1D model. Solid curves correspond to the expression in Eq. (5), while dashed curves correspond to the expression of density in Eq. (8). The circles correspond to points obtained by MC simulation done with a random-sequential update procedure. (b) Average length of the growing lattice $\langle L\rangle$ as a function of time $t$ : The solid curves correspond to the MF expression of Eq. (9). The circles and the squares correspond to $\mathrm{MC}$ simulation results obtained by the random-sequential update procedure and synchronous update procedure, respectively. For all the plots, $\alpha=0.2 \mathrm{~s}^{-1}, \delta=0.2 \mathrm{~s}^{-1}$, lattice spacing $\epsilon=0.4 \mu \mathrm{m}$, the overall growth rate at tip is $0.4 \mu \mathrm{m} \mathrm{s}^{-1}$, and averaging is done for 10000 samples.

the lattice, $V \equiv d\langle L\rangle / d t$, can be expressed as $\frac{d\langle L\rangle}{d t}=\epsilon \rho\langle L\rangle=$ $\epsilon \alpha \exp [K\langle L\rangle / \epsilon]$. With the initial condition of $L$ being 0 at $t=$ 0 , the expression for the average length of the lattice $\langle L\rangle$ as a function of time is

$$
\langle L\rangle=\frac{\epsilon}{|K|} \ln (1+\alpha|K| t) .
$$

The corresponding expression for the mean linear growth rate of the lattice length is

$$
V=\frac{\epsilon \alpha}{1+\alpha|K| t} .
$$

In Fig. 2(b), we show a comparison of the temporal profile of the average length of the growing 1D lattice obtained by approximate analytical means with the temporal profile obtained by MC simulations.

\section{Probability distribution of length of primary hypha}

For the 1D model for growth of the primary fungal hypha, after a fixed interval of time $t$, starting from a single lattice site, the lattice grows to $N$ lattice sites (corresponding to a length $L=\epsilon N)$. Since the process of creation of the defect site is a random process, the length up to which the hypha grows is itself a random variable. Using MC simulations with synchronous update, we simulate the probability distribution of the length of the growing lattice (in terms of the total number of lattice sites $N$ ) after a fixed interval of time. Figure 3(a) displays the probability distribution of $N$ for different value of $t$. For this distribution, the actual value of the peak of the distribution is close to the approximate expression of average length obtained from Eq. (9). Figure 3(b) displays the probability distribution function of the scaled variable, $z=(N-\langle N\rangle) / \sqrt{\langle N\rangle}$, at different times. This distribution tends to a Gaussian distribution. The width of the distribution in terms of scaled variable $z$ decreases very slowly $(\sim 5 \%$, when $t$ increase by one order of magnitude). This implies
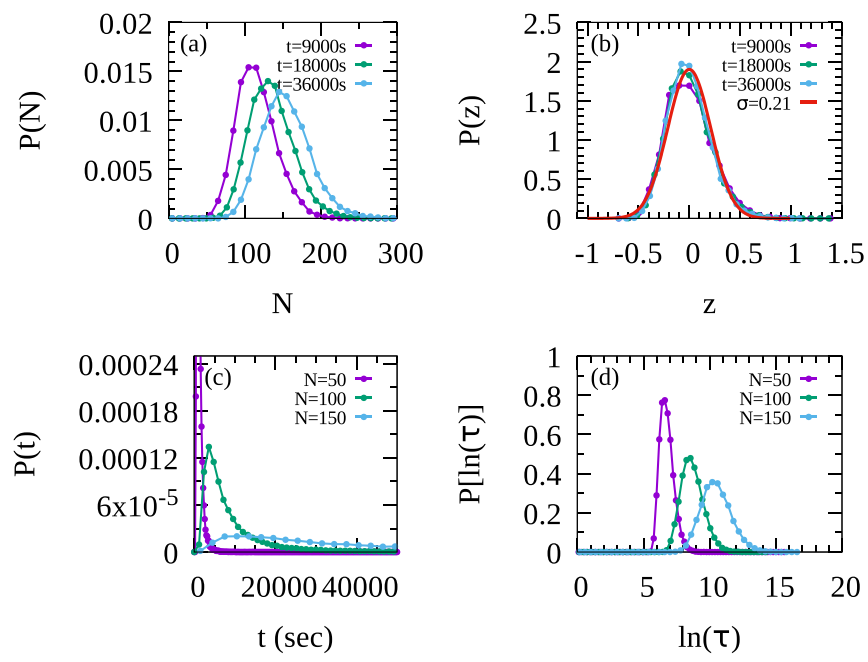

FIG. 3. (a) Probability distribution function of $N$ (corresponding to a length $L=\epsilon N$ of the growing 1D lattice) after time $t$, starting from one lattice site $(N=1)$ at $t=0 \mathrm{~s}$. (b) Probability distribution function in terms of scaled variable $z=(N-\langle N\rangle) / \sqrt{\langle N\rangle}$ at different times. The solid curve corresponds to a Gaussian distribution with $\sigma=0.21$. (c) Probability distribution function of time $t$ required for growth of the lattice from 1 lattice site to $N$ lattice sites. (d) Probability distribution of the $\ln (\tau)$, where $\tau$ is a dimensionless parameter defined as $\tau=t / 1 \mathrm{~s}$. For all the plots, $\alpha=0.2, \gamma=0.9, \delta=0.3$, and the probability distribution functions are obtained by averaging over 5000 samples.

that the relative width of the distribution function of $N$, and equivalently the relative width of the probability distribution function of $L$, decreases more rapidly than $L^{-1 / 2}$. Thus, at late times, the variation of the length of the hypha from the average value is small.

\section{Probability distribution of growth time of hypha}

We look at the probability distribution of time $t$ required for growth of the primary hypha to grow up to a fixed length. Figure 3(c) displays the probability distribution function of time $t$ for different values of total number of lattice sites $N$. The probability distribution function of time is generically a broad distribution, with the most probable value of $t$ significantly different from the mean value of time required for growth of the primary hypha. In Fig. 3(d), we plot the distribution of the $\ln (\tau)$, where $\tau$ is dimensionless and defined as $\tau=t / 1 \mathrm{~s}$. This distribution tends to a Gaussian distribution function, indicating that $P(t)$ tends to a log-normal distribution function.

\section{FEATURES OF SINGLE-COLONY MYCELIUM}

We now focus our attention on the motility and morphology characteristics of a single-colony mycelium. We use the 2D model described in Sec. II B for mimicking the growth processes in single-colony mycelium, which results from elongation and lateral branching of a single primary hypha with a single source of nutrient. The morphology characteristics, e.g., shape and size of the single-colony mycelium, are determined by the parameters $\gamma$, which characterizes 


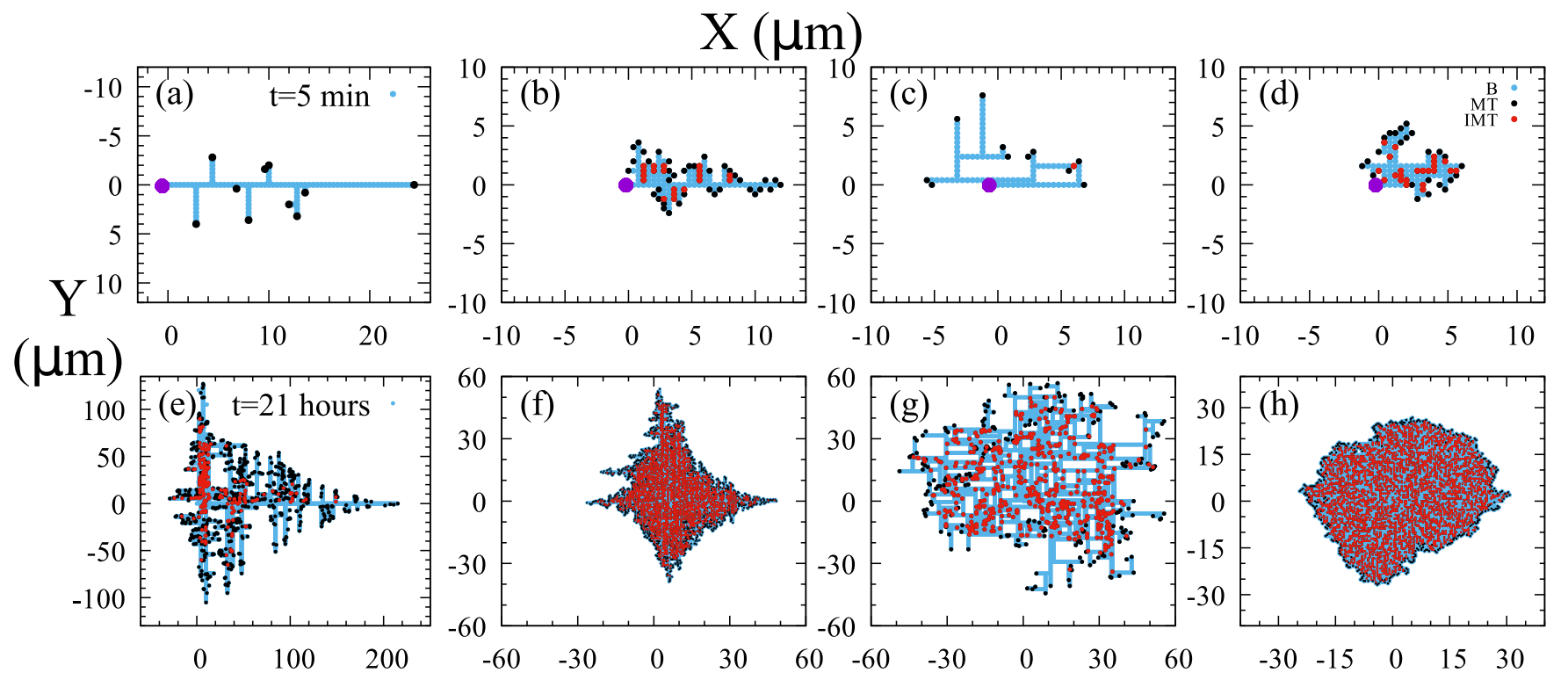

FIG. 4. The morphology of single-colony mycelium (a single sample) at different times $t$, starting from an initial configuration of two lattice sites at $t=0$. The area enclosing each lattice site is a square box with sides of length $0.4 \mu \mathrm{m}$. (a), (e) The morphology at different times ( $t=5$ mins and $t=21$ hours), when $\gamma=0.9, \delta=0.1$, i.e., for low branching rate and low rate of flow of nutrients to secondary branches. (b), (f) The morphologies at different times for $\gamma=0.2, \delta=0.1$, i.e., for high branching rate and low rate of flow of nutrients to secondary branches. (c), (g) The morphologies at different times for $\gamma=0.9, \delta=0.8$, i.e., for low branching rate and high rate of flow of nutrients to secondary branches. (d), (h) The morphologies at different times for $\gamma=0.2, \delta=0.8$, i.e., for high branching rate and high rate of flow of nutrients to secondary branches. For all cases, $\alpha=0.2$. The mobile tip sites (MT), immobile tip sites (IMT), and bulk sites (B) of the growing colony are indicated in each panel.

the propensity for branching in the hypha, and $\delta$, which is a parameter that characterizes the nutrient flow rate to the secondary branch and input rate of nutrients $\alpha$. Depending on the choice of these parameters, the morphology characteristics can vary significantly. For experiments with Neurospora crassa, the experimentally observed growth rates of hypha are in the range of $20-30 \mu \mathrm{m} / \mathrm{min}$ [31,32]. Consistent with this observation, we set the overall growth rate in our model system to $0.4 \mu \mathrm{m} / \mathrm{s}$ and choose a lattice spacing $\epsilon=0.4 \mu \mathrm{m}$, which corresponds to an overall growth rate of $1 \mathrm{~s}^{-1}$. This would also imply that the particles in the bulk of the lattice are hopping, with rate $0.4 \mu \mathrm{m} \mathrm{s}^{-1}$ corresponding to hop rate of $1 \mathrm{~s}^{-1}$ that we have set for our model. This choice of hopping rate is comparable to the typical motor velocity of kinesin-1 motors, whose velocity is $\sim 0.6 \mu \mathrm{m} \mathrm{s}^{-1}$ [31]. Figure 4 displays some of the resultant morphologies and their evolution over time, for different sets of parameters. As would be expected, when both the branching rate $1-\gamma$ and nutrient flow rate to the secondary branch $\delta$ are low, the lateral growth of hyphae is small compared to the longitudinal growth along the direction of growth of the primary hypha, in the initial phase of evolution of the single colony [Fig. 4(a)] and it continues to persist after 21 hours [Fig. 4(e)]. Figures 4(b) and 4(f) show the temporal evolution of the morphology when the branching rate is high and rate of nutrient supply to secondary branches is low, while Figs. 4(c) and $4(\mathrm{~g})$ show the typical morphology when the branching rate is low while the rate of supply to the secondary branches is high. When both the branching rate and nutrient flow rate to the branches are high, asymmetry of growth along the longitudinal and lateral directions is virtually absent and the single-colony mycelium tends to a radially symmetric configuration about its center of mass at late times [Fig. 4(h)].

The process of branching in the hyphae has the effect of creating new tip sites $(\mathrm{T})$, which in turn serve as seeds for further elongation and branching process of the mycelium. However, as described in Sec. II B, the tips can elongate or branch only if the adjacent space is not occupied by the single-colony mycelium. For the case where the branching rate and $\delta$ is high, not only would there be numerous branches which would be created in the mycelium, but there would be a tendency of the growth direction of the tips to change and consequently be obstructed by the preexisting mycelium. Thus, over the course of evolution of the morphology, many of the growing tips (T) would become immobile or jammed, i.e., these tips would not serve as either elongation or branching site. Along with the different morphologies obtained for the different values of control parameters $\gamma$ and $\delta$, Fig. 4 also shows the immobile and mobile tip sites of the mycelium after time $t$ has elapsed starting from a single hypha of unit length.

\section{A. Motility behavior of center of mass}

In Fig. 5(a), we display the temporal behavior of the center of mass of the growing lattice network (averaged over different samples). Increasing the branching rate $1-\gamma$ and/or $\delta$ has the effect of impeding the motility of the center of mass of the single-colony mycelium. As expected for early times, for low $\delta$ and low branching rates, $\sqrt{\left\langle R_{c m}^{2}\right\rangle}$ shows a linear dependence on the $\tau$. The log-log plot in Fig. 5(b) suggests that $\sqrt{\left\langle R_{c m}^{2}\right\rangle} \sim t^{q}$ at later times. For all the cases, we find that the exponent $q<1 / 2$ indicates subdiffusive behavior of the 

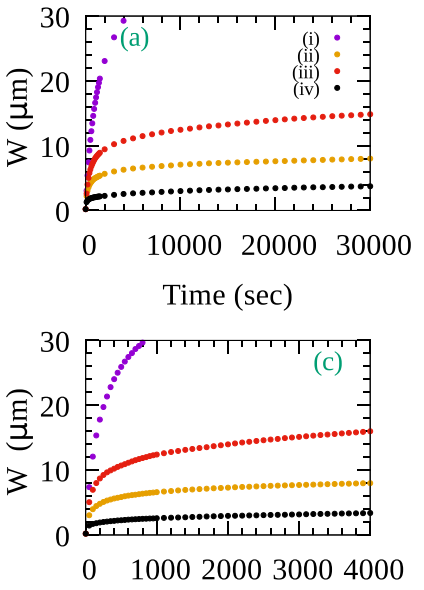

$\mathrm{N}$
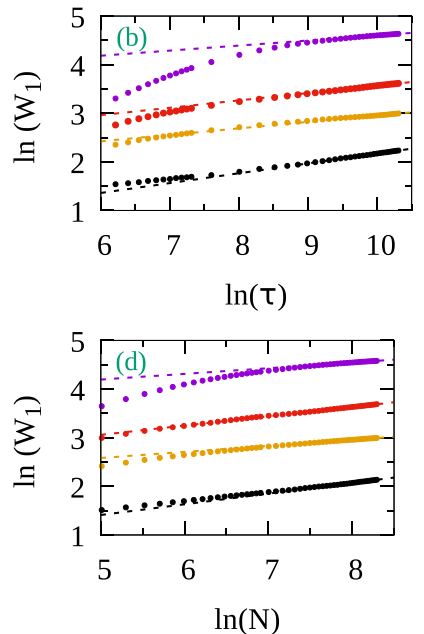

FIG. 5. Behavior of center of mass $W \equiv \sqrt{\left\langle R_{c m}^{2}\right\rangle}$ for four different parameter values: (i) $\gamma=0.9, \delta=0.1$, (ii) $\gamma=0.2, \delta=0.1$, (iii) $\gamma=0.9, \delta=0.8$, and (iv) $\gamma=0.2, \delta=0.8$. For all cases, $\alpha=0.2$ and $\epsilon=0.4 \mu \mathrm{m}$. These sets of parameter values are the same as in Figs. 4(a)-4(d), respectively. (a) The plot of $W$ vs $t$. (b) The plot of $\ln \left(W_{1}\right)$ vs $\ln (\tau)$, where $W_{1}=W / \epsilon$ is the dimensionless center of mass, and $\tau=t / 1 \mathrm{sec}$ is the dimensionless time. The least-squaresfitted straight line to the data points at later times corresponds to a slope of (i) $q=0.10$, (ii) $q=0.13$, (iii) $q=0.15$, and (iv) $q=0.20$, where $W_{1} \sim \tau^{q}$. (c) Plot of $W$ vs $N$. (d) Plot of $\ln \left(W_{1}\right)$ vs $\ln (N)$ : The least-squares-fitted straight line to the data points at large $N$ corresponds to a slope of (i) $\beta=0.12$, (ii) $\beta=0.12$, (iii) $\beta=0.19$, and (iv) $\beta=0.22$, where $W_{1} \sim N^{\beta}$. Averaging is done over 100 samples.

center of mass for the regime of the time window that we have probed. This power-law behavior of the motility of the center of mass of the $2 \mathrm{D}$ colony is in contrast to the motility behavior of the center of mass for just the primary hypha (1D model), where indeed the center of mass of the primary hypha exhibits a logarithmic dependence on time. In order to check whether the motility behavior of the center of mass is better described by a power-law dependence on time than a logarithmic dependence on time, we make a numerical comparison of the two scenarios using the data corresponding to the temporal growth of the center of mass. In particular, we compare the quality of the fitted straight line corresponding to a power-law dependence of $\sqrt{\left\langle R_{c m}^{2}\right\rangle}$ on time with a straight line obtained by fitting $\sqrt{\left\langle R_{c m}^{2}\right\rangle}$ with logarithmic dependence on time. Based on the quality of fit to the data of the motility of the center of mass, we can infer that the motility behavior of the center of mass is better described by a power-law dependence on time (Fig. 11; see the Appendix). It is worthwhile to point out that there is one crucial difference between the growth process of the $2 \mathrm{D}$ colony and the growth process of the individual hypha. While, for the growth process of individual hypha, the particle number in bulk of the 1D lattice is not conserved due to loss from defect sites, which in turn leads to logarithmic temporal growth of the length for the primary hypha, for the 2D colony growth process, there is always particle-number conservation in the bulk, with the growth happening at multiple tips.

Figure 5(c) shows the dependence of $\left\langle R_{c m}^{2}\right\rangle$ on $N$. From the log-log plot displayed in Fig. 5(d) for different sets of
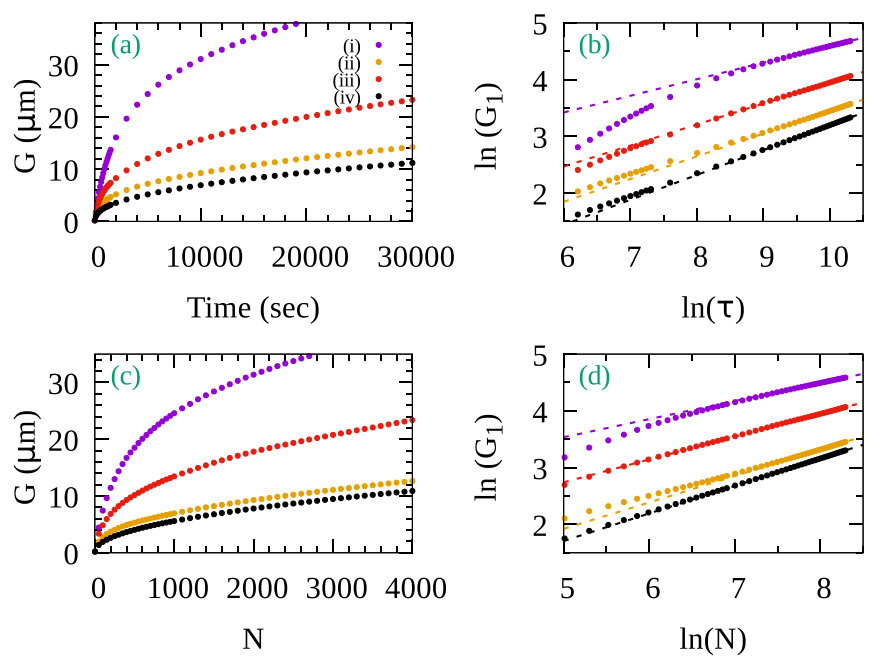

FIG. 6. Behavior of radius of gyration $G=\sqrt{\left\langle R_{G}^{2}\right\rangle}$ for four different parameter values (same as Fig. 5). (a) The plot of $G$ vs $t$. (b) Plot of $\ln \left(G_{1}\right)$ vs $\ln (\tau)$, where $G_{1}=G / \epsilon$ is the dimensionless radius of gyration, and $\tau=t / 1 \mathrm{sec}$ is the dimensionless time. The least-squares-fitted straight line to the data points at later times corresponds to a slope of (i) $\phi=0.29$, (ii) $\phi=0.40$, (iii) $\phi=0.37$, and (iv) $\phi=0.44$, where $G_{1} \sim \tau^{\phi}$. (c) Plot of $G$ vs $N$. (d) Plot of $\ln \left(G_{1}\right)$ vs $\ln (N)$ : The least-squares-fitted straight line to the data points at large $N$ corresponds to a slope of (i) $v=0.32$, (ii) $v=0.47$, (iii) $v=0.40$, and (iv) $v=0.48$, where $G_{1} \sim N^{v}$. Averaging is done over 100 samples.

input parameters, we see that for sufficiently large size $N$, $\sqrt{\left\langle R_{c m}^{2}\right\rangle} \sim N^{\beta}$.

\section{B. Size of the colony}

The radius of gyration $R_{g}$ is one measure which can be used to characterize the typical size of the single-colony mycelium. In order to understand the growth characteristics of the size of the single colony, we look at the temporal behavior of $\sqrt{\left\langle R_{g}^{2}\right\rangle}$. Figure 6(a) displays the temporal evolution of $\left\langle R_{g}^{2}\right\rangle$. From the log-log plot in Fig. 6(b), we can infer that at later times, $\left\langle R_{g}^{2}\right\rangle \sim t^{\phi}$ with $\phi<1 / 2$. Figure 6(c) displays the variation of $\left\langle R_{g}^{2}\right\rangle$ with $N$ for different sets of input parameters. Assuming a power-law dependence of $\sqrt{\left\langle R_{G}^{2}\right\rangle}$ on $N$ of the form $\left\langle R_{g}^{2}\right\rangle \sim$ $N^{2 v}$, from the log-log plot in Fig. 6(d) we extract the exponent $v$ from the slope of the least-squares-fitted straight line. For the range of $N$ considered for our simulations, the exponent $v$ varies with the chosen input parameters $\delta$ and $\gamma$. Typically, the effect of lowering the branching rate has the effect of increasing $\left\langle R_{G}^{2}\right\rangle$ for a given $N$. When the nutrient supply rate $\delta$ is made high while the branching rate is lowered, the resultant colony becomes more porous, with less dense filling of space. This attribute of the colony is demonstrated by the morphology of the colony shown in Fig. 4(g). This may be understood as follows: When the branching rate is low, very few branches are created. However, the secondary branches are the ones which grows faster. Subsequent branching of these secondary branches also eventually results in the formation of a closed loop, which arrests further growth of the branches locally due to obstruction. The size of this loop determines the porousness 


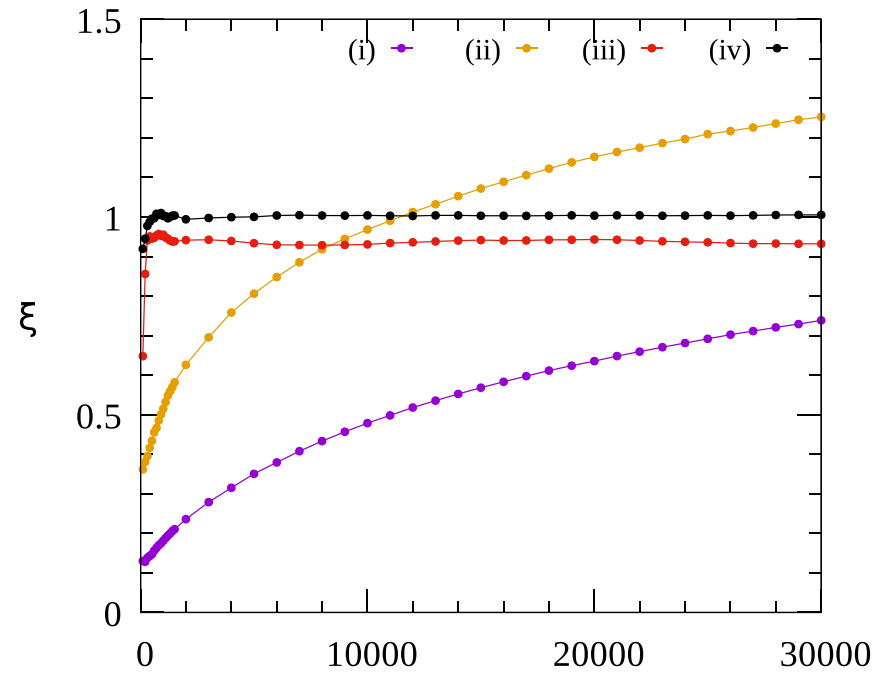

Time (sec)

FIG. 7. Temporal evolution of the aspect ratio $\xi=\sqrt{\left\langle Y_{g}^{2}\right\rangle /\left\langle X_{g}^{2}\right\rangle}$ : Plot of $\xi$ vs $t$ for four different parameter values (same as Fig. 5). Averaging is done over 100 samples.

of the colony. Since the size of these closed loops increases when the branching rate is decreased and $\delta$ is increased, the resultant overall morphology becomes more porous. When both the branching rate is high and $\delta$ is high, it would be expected that the porousness would be low and the growth of the colony would be a space-filling process. Such a scenario is observed for case (iv), for which $\gamma=0.2, \delta=0.8$. The typical configuration of the colony for this particular choice of parameters is illustrated in Fig. 4(h). For this case, the corresponding value of the exponent $v \simeq 0.5$ in the large- $N$ limit. This value of the exponent $v$ is the same as that of the $2 \mathrm{D}$ Eden model for growth in the large- $N$ limit $[39,43]$. Further, for the large- $N$ limit, $\left\langle R_{G}^{2}\right\rangle \rightarrow N / 2 \pi$, which is again similar to the results obtained for the 2D Eden model [43]. In fact, any 2D growth process which incorporates the excluded volume effect and allows for maximum occupancy of 1 per lattice site would necessarily have to satisfy the condition $\left\langle R_{g}^{2}\right\rangle \geqslant N / 2 \pi$. This condition arises purely due to geometric constraint. The equality holds for the case of complete space filling on a circle. Except for case (iv), for which $\left\langle R_{g}^{2}\right\rangle$ approaches the limiting value of $N / 2 \pi$, for the other three cases, $\left\langle R_{g}^{2}\right\rangle>N / 2 \pi$ [Fig. 6(c)] up to $N=4000$. Further, the value of the exponent $v<0.5$ for the other three cases, which implies that the value of the exponent $v$ characterizing the power-law behavior of the radius of gyration (up to $N=4000$ ) is different from the exponent $v$ which characterizes the truly "large- $N$ " limiting behavior of $\left\langle R_{g}^{2}\right\rangle$.

\section{Shape characteristics of the colony}

In order to characterize the asymmetry of the morphology of the single colony, we define aspect ratio $\xi=\sqrt{\left\langle Y_{g}^{2}\right\rangle /\left\langle X_{g}^{2}\right\rangle}$. $\xi$ is a measure of the relative growth in the lateral direction vis-a-vis the extension along the original direction of growth of the primary hypha. Figure 7 shows the temporal behavior of the aspect ratio. For very late times, the aspect ratio is expected to converge to 1 . Indeed, when the branching rate is low and nutrient supply rate to the secondary branches is high, as in case (iv), the aspect ratio of the colony tends to 1 very rapidly, which corresponds to a typical morphology of the colony shown in Fig. 4(h). However, when the branching rate is low and $\delta$ is also low, for early times, the lateral extension of the colony (perpendicular to the initial growth direction of the original hypha) is much less compared to the growth along the original direction along $x$. Thus, even after more than 8 hours of growth, the aspect ratio is much lower than 1, approaching the "late" time limit of $\xi=1$ slowly. Interestingly, for a range of intermediate times (up to 30000 secs) considered in the simulation, when the branching rate is very high while the nutrient supply rate to the branches is low, as in case (ii), the aspect ratio $\xi$ even exceeds 1 (Fig. 7). This may be qualitatively understood as follows: When the branching rate is high while $\delta$ is low, from the primary hypha, many lateral branches (along the $y$ axis) would be generated and the typical distances between these lateral branches would be relatively small. Over the course of evolution of the colony, these lateral branches would be the sites for secondary branches along the $x$ axis - the direction of growth of the original primary hypha. However, the growth of these secondary branches would be arrested by the frequent lateral branches arising due to the high rate of branching. This is turn would lead to a situation where the overall growth along the $x$ direction (except the primary branch) would be slowed down compared to the unhindered growth of the secondary branches along the lateral direction along the $y$ axis. Such growth process results in a typical morphology of the colony shown in Fig. 4(f) at intermediate times. However, even for this case, at later time ( $\sim 130$ hours), $\xi$ converges to 1 .

\section{Growth sites of the colony}

For the 2D model for the growing colony that we have considered, growth occurs only at the tip sites. While the elongation process shifts the position of the tip site, the branching process results in the creation of new tips. Over the course of evolution of the morphology of the colony, many of the tip sites cease to be sites of growth and become immobile as they are obstructed by the part of the preexisting colony. Figure 8 displays the temporal behavior of $Q$, which is the ratio of the number of mobile tips $N_{m}$, with total number of tips $N_{T}$ in a single colony. $Q$ decreases with the passage of time as the colony size increases. Expectedly, the rate of decrease of $Q$ is faster for the case when the branching rate is high and the nutrient supply rate to secondary branches is also high [case (iv)] in comparison to the case when both the branching rate and $\delta$ are low [case (i)]. In fact, at "late" times and "large"- $N$ limit, the mobile tips would reside typically on the outer perimeter of the colony, while the immobile tips would be part of the bulk [Fig. 4(h)]. As the size of the colony grows, the ratio of the number of sites on the outer perimeter with the total number of sites decreases and, consequently, we would expect the decrease in $Q$ as we observe in the simulations. In order to quantify the typical scaling behavior exhibited by these mobile tips in the large- $N$ limit, we specifically focus on the case where the branching rate is high and for which $\delta$ is 


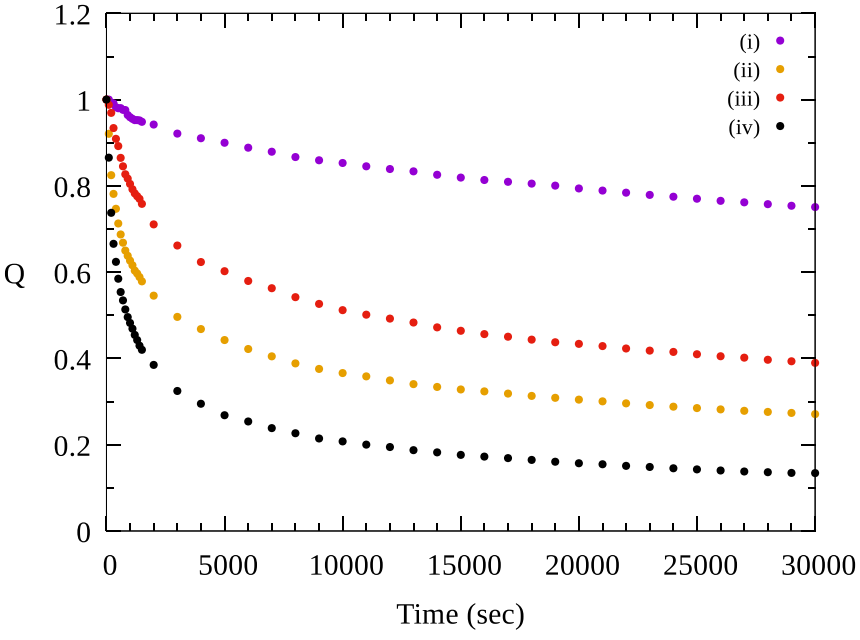

FIG. 8. Temporal behavior of the mobile tip ratio $Q \equiv N_{m} / N_{T}$, where $N_{m}$ is the number of mobile tips and $N_{T}$ is the total number of tips. Variation of $Q$ for four different parameter values (same as Fig. 5) is shown. Averaging is done over 100 samples.

low, since for this case convergence to large- $N$ limit behavior is relatively faster. We define $S$ as the ratio of the number of mobile tips, $N_{m}$, with the total number of lattice sites of the colony $N$. In Fig. 9(a), we show the variation of $S$ with the radius of gyration $R_{g}(N)$. From the corresponding log-log plot in Fig. 9(b), we can infer $S \sim R_{g}^{-1}$. This particular scaling behavior may be understood as follows: For the large- $N$ limit, the mobile tips reside on the outer perimeter of the colony. Further, if the perimeter is an euclidean surface, then the ratio of the perimeter length with total area should scale inversely
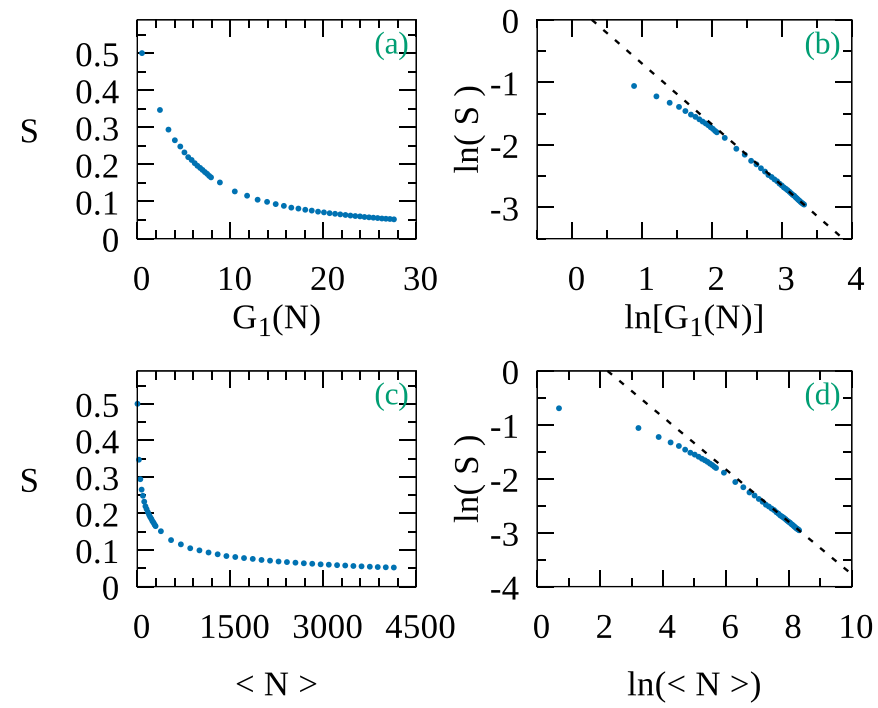

FIG. 9. Scaling behavior of $S \equiv\left\langle N_{m}\right\rangle /\langle N\rangle$ : (a) Variation of $S$ with scaled radius of gyration $G_{1}$. (b) Plot of $\ln (S)$ vs $\ln \left(G_{1}\right)$. The least-squares-fitted straight line to the data points at large $N$ corresponds to a slope of $\theta=-1.03$, where $S \sim G_{1}^{\theta}$. (c) Variation of $S$ with $\langle N\rangle$. (d) Plot of $\ln (S)$ vs $\ln (\langle N\rangle)$. The least-squares-fitted straight line to the data points at large $N$ corresponds to a slope of $\eta=-0.49$, where $S \sim N^{\eta}$. Here, $\gamma=0.2, \delta=0.8$, and $\alpha=0.2$. Averaging is done over 100 samples.
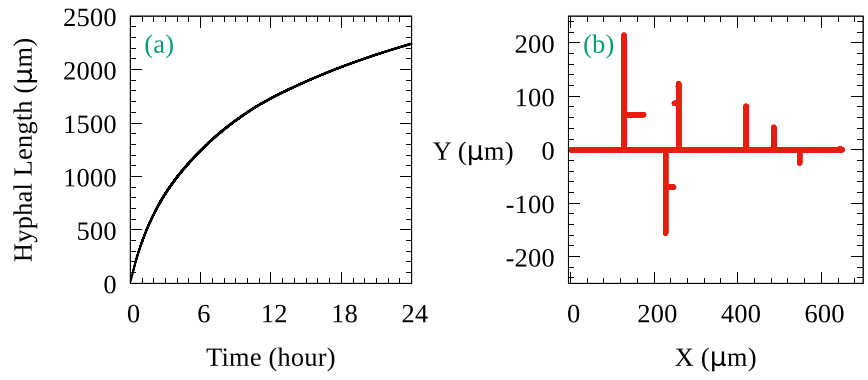

FIG. 10. (a) Average length of lattice $\langle L\rangle$ as a function of time $t$ : The solid curves correspond to MC simulation results for a $1 \mathrm{D}$ model. Here, $\alpha=0.4 \mathrm{~s}^{-1}, \delta=0.2 \mathrm{~s}^{-1}, \gamma=0.998 \mathrm{~s}^{1}$, lattice spacing $\epsilon=0.4 \mu \mathrm{m}$, and velocity at the growing tip is $0.4 \mu \mathrm{m} \mathrm{s}^{-1}$. For this choice of parameters, the hyphal growth characteristics observed experimentally for fungal hypha of Rhizopus oligoporous [9,50] match reasonably well with the MC simulation results. (b) The 2D morphology after 5 hours obtained for $\alpha=0.4 \mathrm{~s}^{-1}, \delta=0.2 \mathrm{~s}^{-1}$, $\gamma=0.995, \epsilon=0.4 \mu \mathrm{m}$, and velocity at the tip is $0.4 \mu \mathrm{m} \mathrm{s}^{-1}$. This morphology is similar to the morphology of the single-colony hypha obtained in experiments with Neurospora crassa [32].

with $R_{g}$ and, consequently, $S$ itself scales inversely with the radius of gyration. Since for this case, from our simulations, we know that $R_{g} \sim N^{1 / 2}$ [Fig. 6, case (iv)], it would be expected that $S \sim N^{-1 / 2}$. Figure 9(c) displays the variation of $S$ with $N$. The corresponding log-log plot displayed in Fig. 9(d) indeed confirms this scaling behavior.

\section{COMPARISON WITH EXPERIMENTS}

The only control parameters of the 2D minimal model that we have presented are the entry rate $\alpha$, the branching rate $1-$ $\gamma$, and nutrient supply rate to secondary branches $\delta$. In order to make connection with the morphological features observed in experiments, we check whether, by calibrating these three input parameters, the model is able to reproduce quantitative measures associated with the growth characteristics of fungal hyphae. Consistent with experiments with fungal hyphae of Neurospora crassa, we choose overall hyphal extension rate $0.4 \mu \mathrm{m} / \mathrm{s}[31,32]$ and set the lattice spacing $\epsilon=0.4 \mu \mathrm{m}$. This would correspond to an overall growth rate of 1 lattice unit per second. For experiments with Neurospora crassa, the experimentally observed growth rates of hypha are in the range $20-30 \mu \mathrm{m} / \mathrm{min}$ [31,32]. We choose a particle hopping rate of $1 \mathrm{~s}^{-1}$. This would also imply that the particles in the bulk of the lattice are hopping with rate $0.4 \mu \mathrm{m} \mathrm{s}^{-1}$. This choice of hopping rate compares reasonably with the typical motor velocity of kinesin-1 motors [31].

In Fig. 10(a), we plot the temporal behavior of the average length of the primary hypha, using MC simulations of the 1D model discussed in Sec. II A for a particular choice of input parameters. This profile quantitatively reproduces reasonably well the temporal profile obtained experimentally for the hyphal growth of Rhizopus oligoporous [9,50].

In Fig. 10(b), we show the morphology of a single sample obtained by $\mathrm{MC}$ simulations of the $2 \mathrm{D}$ model discussed in Sec. II B for a particular choice of parameters $\alpha, \gamma$, and $\delta$ after 5 hours of growth. This simulated morphology compares 
well with the morphology observed in experiments with $\mathrm{Neu}$ rospora crassa after 5 hours of growth [32].

\section{CONCLUSIONS AND DISCUSSION}

In this article, we have discussed a minimal driven lattice gas model which generates the morphological characteristics associated with single-colony mycelium arising from the growth and branching process of fungal hyphae, which is fed by a single source of nutrients. While the $1 \mathrm{D}$ model describes the growth characteristics of the primary hypha, the 2D model provides a description of the entire single-colony mycelium that is generated by the elongation and branching process of the fungal hyphae.

The 1D model predicts a spatial profile of particles which is exponential along the direction of growing primary hypha, and a length of the primary hypha which grows logarithmically with time. Our MC simulation results show that the sample-to-sample relative fluctuation of the average length of the primary hypha at late times is small. However, we find that the probability distribution of the time required to grow to a specified length is broad and it tends to a log-normal distribution at late times. Although we have not been able to explicitly derive an explicit analytical from the distribution function of the growth time, we have been able to show that the analytical form of the survival probability of the particle to reach distance $L$ along the primary lattice without getting lost from the primary lattice is a log-normal distribution. As an aside, it may be noted that this kind of log-normal distribution with its implication of huge sample-to-sample variation of the measured growth time is also seen in the context of a probability distribution function of a tangent-tangent correlation function of a random heteropolymer [51] and spin-spin correlation function for a random Ising spin chain [52].

Using MC simulations, we generate the different types of 2D morphologies of single-colony mycelium. We find a wide variability of motility, size, and shape characteristics of the 2D morphology depending on the input parameters, e.g., branching rate $1-\gamma$, nutrient flow rate to secondary branches $\delta$, and input rate of nutrients $\alpha$. The center of mass $R_{c m}$ and the radius of gyration $R_{g}$ of the simulated colony exhibit a subdiffusive behavior, at later times. Our analysis also reveals a power-law dependence of $R_{g}$ on $N$ of the form $R_{g} \sim N^{\nu}$. When both the branching rate is high and $\delta$ is high, for sufficiently high $N$, the behavior of $R_{g}$ as a function of $N$ is characterized by an exponent which seems to converge to $v \sim 0.5$, which is similar to the value of the exponent that characterizes the growth process of an Eden cluster in the large- $N$ limit $[39,43]$. A more detailed investigation is needed to confirm whether the growth process of our 2D model, in the limit of high branching rate and high $\delta$, belongs to the same universality class as that of the Eden model $[38,39,43]$. More generally, it would be interesting to study the similarities and the differences of the asymptotic characteristics of the growing colony at large- $N$ and late-time limit for this model
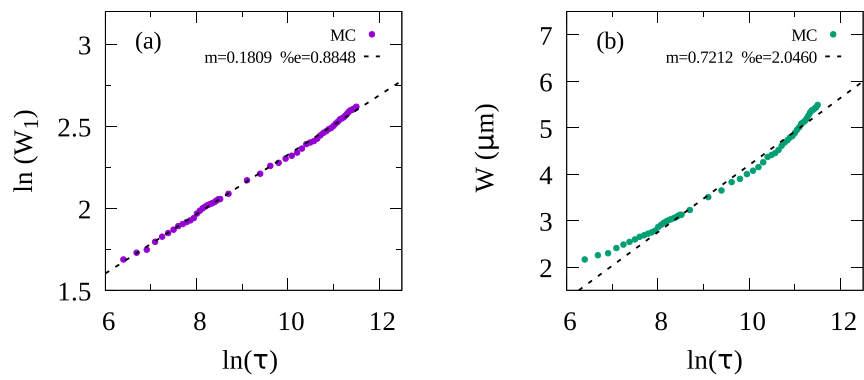

FIG. 11. Temporal behavior of center of mass (W). (a) Powerlaw fit: Plot of $\ln \left(W_{1}\right)$ vs $\ln (\tau)$, where $W_{1}=W / \epsilon$ is the dimensionless center of mass and $\tau=t / 1 \mathrm{sec}$ is the dimensionless time. The leastsquares-fitted straight line to the data points corresponds to a slope of $m=0.18$ where $W_{1} \sim \tau^{m}$. (b) Straight-line fit for $\sqrt{\left\langle R_{c m}^{2}\right\rangle}$ vs $\ln (\tau)$ : The least-squares-fitted straight line to the data points corresponds to a slope of $m=0.72$. For both plots, $\alpha=0.2, \gamma=0.2$, and $\delta=0.8$. These parameters are the same as in case (iv) in Fig. 5. Averaging is done over 10 samples.

with other lattice animal models of growth $[39,41,43]$ and polymerization process of branched polymers [35-37,45,46].

We find that by suitable calibration of the parameters of the model, we are able to quantitatively reproduce the observed experimental growth characteristics of the primary hypha of Rhizopus oligoporous $[9,50]$, and we are also able to replicate the morphology characteristics of the single-colony mycelium of Neurospora crassa observed in experiments [32].

While, in this article, we have restricted ourselves to analyzing the morphology characteristics of the colony for which the tip site stops growing and branching when it encounters obstruction due to the presence of the already grown lattice, it would interesting to study the morphology characteristics of the colony for the situation in which parts of the growing network are allowed to overlap over each other to form a layered mesh.

\section{ACKNOWLEDGMENTS}

Financial support is acknowledged by S.M. for SERB Project No. EMR/2017/001335. S.M. would like to thank D. Dhar (IISER, Pune) and Ignacio Pagonabarraga (CECAM, Switzerland) for useful discussions and suggestions.

B.S. carried out simulations and analyzed data. S.K. carried out simulations for the 1D model. S.M. designed the study, analyzed the data, and wrote the manuscript.

\section{APPENDIX: TEMPORAL BEHAVIOR OF CENTER OF MASS}

In Fig. 11, we show a comparison of the least-squaresfitted straight line corresponding to a power-law dependence of $\sqrt{\left\langle R_{c m}^{2}\right\rangle}$ on time $t$ with a straight line obtained by fitting $\sqrt{\left\langle R_{c m}^{2}\right\rangle}$ with $\ln (t)$. As can be seen from the quality of fit, the power-law fit appears to be more appropriate compared to fitting data with $\ln (t)$.
[1] J. W. Deacon, Fungal Biology, 4th ed. (Blackwell, Oxford, 2006).
[2] J. E. Galagan, M. R. Henn, Li-Jun Ma, C. A. Cuomo, and B. Birren, Genome Res. 15, 1620 (2005). 
[3] B. Alberts et al., Molecular Biology of the Cell, 6th ed. (Garland Science, New York, 2007).

[4] S. Bartnicki-Garcia, F. Hergert, and G. Gierz, Protoplasma 153, 46 (1989).

[5] S. H. Tindermann, N. Kern, and B. M. Mulder, J. Theor. Biol. 238, 937 (2006).

[6] F. A. Davidson, Fungal. Biol. Rev. 21, 30 (2007).

[7] J. I. Prosser and A. P. J. Trinci, J. Gen. Microbiol. 111, 153 (1979).

[8] K. E. P. Sugden, M. R. Evans, W. C. K. Poon, and N. D. Read, Phys. Rev. E 75, 031909 (2007).

[9] W. Balmant, M. H. Sugai-Guerios, J. H. Coradin, N. Krieger, A. F. Junior, and D. A. Mitchell, PLoS ONE 10, e0120307 (2015).

[10] G. P. Boswell and F. A. Davidson, Fungal Biol. Rev. 26, 20 (2012).

[11] M. R. Islam, G. Tudryn, R. Bucinell, L. Schadler, and R. C. Picu, Sci. Rep. 7, 13070 (2017).

[12] T. Chou and D. Lohse, Phys. Rev. Lett. 82, 3552 (1999).

[13] C. T. MacDonald, J. H. Gibbs, and A. C. Pipkin, Biopolymers 6, 1 (1968).

[14] A. Parmeggiani, T. Franosch, and E. Frey, Phys. Rev. Lett. 90, 086601 (2003).

[15] A. Parmeggiani, T. Franosch, and E. Frey, Phys. Rev. E 70, 046101 (2004).

[16] S. Muhuri and I. Pagonabarraga, Phys. Rev. E 82, 021925 (2010).

[17] Y. Aghababaie, G. I. Menon, and M. Plischke, Phys. Rev. E 59, 2578 (1999).

[18] S. Chandel, A. Chaudhuri, and S. Muhuri, Europhys. Lett. 110, 18002 (2015).

[19] K. E. P. Sugden and M. R. Evans, J. Stat. Mech. (2007) P11013.

[20] S. Muhuri, Europhys. Lett. 101, 38001 (2013).

[21] L. B. Shaw, R. K. P. Zia, and K. H. Lee, Phys. Rev. E 68, 021910 (2003).

[22] G. Lakatos, J. O’Brien, and T. Chou, J. Phys. A 39, 2253 (2006).

[23] R. Juhasz and L. Santen, J. Phys. A 37, 3933 (2004).

[24] G. M. Schutz, J. Phys. A 36, R339 (2003).

[25] B. Derrida, E. Domany, and D. Mukamel, J. Stat. Phys. 69, 667 (1992).
[26] M. R. Evans, D. P. Foster, C. Godreche, and D. Mukamel, Phys. Rev. Lett. 74, 208 (1995).

[27] M. R. Evans, D. P. Foster, C. Godreche, and D. Mukamel, J. Stat. Phys. 80, 69 (1995).

[28] V. Popkov and G. M. Schutz, J. Stat. Phys. 112, 523 (2003).

[29] M. R. Evans, Y. Kafri, H. M. Koduvely, and D. Mukamel, Phys. Rev. Lett. 80, 425 (1998).

[30] H. E. Gruen, Plant Physiol. 34, 158 (1959).

[31] G. Steinberg, Eukaryotic Cell 6, 351 (2007).

[32] T. V. Potapova, L. Yu. Boitsova, S. A. Golyshev, and A. Ya. Dunina-Barkovskaya, Cell Tissue Biol. 58, 634 (2016).

[33] S. Seiler, M. Plamann, and M. Schliwa, Curr. Biol. 9, 779 (1999).

[34] R. R. Mourino-Pérez, R. W. Roberson, and S. Bartnicki-García, Fungal. Gen. Biol. 43, 389 (2006).

[35] M. Daoud and J. F. Joanny, J. Phys. 42, 1359 (1981).

[36] A. Rosa and R. Everaers, J. Chem. Phys. 145, 164906 (2016).

[37] R. Everaers, A. Y. Grosberg, M. Rubinstein, and A. Rosa, Soft Matter 13, 1223 (2017).

[38] M. Eden, in Proceedings of Fourth Berkeley Symposium in Mathematics, Statistics and Probability, edited by J. Neyman (University of California Press, Berkeley, 1961).

[39] D. Dhar and R. Ramaswamy, Phys. Rev. Lett. 54, 1346 (1985).

[40] S. S. Manna and D. Dhar, Rhy. Rev. E 54, R3063 (1996).

[41] H. Hsu, W. Nadler, and P. Grassberger, J. Phys. A 38, 775 (2005).

[42] M. Plischke and Z. Rácz, Phys. Rev. Lett. 53, 415 (1984).

[43] Z. Racz and M. Plischke, Phys. Rev. A 31, 985 (1985).

[44] F. Family and T. Vicsek, J. Phys. A 18, L75 (1985).

[45] T. C. Lubensky and J. Isaacson, Phys. Rev. A 20, 2130 (1979).

[46] T. C. Lubensky and J. Isaacson, J. Phys. 41, L469 (1980).

[47] N. Rajewsky, L. Santen, A. Schadschneider, and M. Schreckenberg, J. Stat. Phys. 92, 151 (1998).

[48] M. Schreckenberg, A. Schadschneider, K. Nagel, and N. Ito, Phys. Rev. E 51, 2939 (1995).

[49] L. G. Tilstra and M. H. Ernst, J. Phys. A 31, 5033 (1998).

[50] M. Nopharatana, D. A. Mitchell, and T. Howes, Biotechnol. Bioeng. 84, 71 (2003).

[51] S. Muhuri and M. Rao, J. Stat. Mech. (2010) P02005.

[52] B. Derida and H. Hilhorst, J. Phys. C 14, L539 (1981). 